\title{
Modified PTS with FECs for PAPR Reduction of OFDM Signals
}

\author{
P. Mukunthan \\ Department of ECE \\ Pondicherry Engineering College \\ Puducherry, India.
}

\author{
P.Dananjayan \\ Department of ECE \\ Pondicherry Engineering College \\ Puducherry, India.
}

\begin{abstract}
Partial transmit sequence (PTS) is an attractive technique for PAPR reduction without distortion, but to obtain preferable PAPR performance it needs many inverse fast Fourier transforms (IFFTs), which results in high computational complexity. In order to reduce the complexity, modified PTS technique, with real and imaginary parts, separately multiplied with phase factors is considered in this paper. To reduce PAPR further the forward error-correcting codes (FECs) such as Golay codes and Turbo codes are employed in the modified PTS radix FFT, where, the PAPR is jointly optimized in both the real and imaginary part. The simulation results show that the combined FEC with modified PTS technique significantly provides better PAPR reduction with reduced computational complexity compared to ordinary PTS with FEC.
\end{abstract}

\section{General Terms}

Orthogonal frequency division multiplexing (OFDM), peak-toaverage power ratio (PAPR), partial transmit sequence (PTS). Keywords Golay complementary sequences, Reed-Muller code, Turbo code.

\section{INTRODUCTION}

Orthogonal Frequency Division Multiplexing (OFDM) has been widely used in modern wireless communications because of its inherent robustness to frequency selective fading channels [1]. Due to this advantage, it finds applications in Digital Audio and Video Broadcasting (DAB and DVB-T), Digital Subscriber Lines (x-DSL), and wireless local area network. One of the major drawbacks of any OFDM system is that the signal has a nonconstant envelope exhibiting peaks that exceeds the mean power, and the signal is said to have a high peak-to-average power ratio (PAPR). To prevent spectral growth of the OFDM signal in the form of intermodulation among subcarriers and out-of-band radiation [2], the transmit amplifier must be operated in its linear region. Amplifiers with large linear range are expensive and this can increase the cost of the implementation of OFDM. To overcome such effects, many PAPR reduction techniques such as distortion, distortionless or coding have been proposed for OFDM signals [3-12]. Distortionless techniques such partial transmit sequence (PTS) [13] and selective mapping (SLM) [14] can improve the PAPR statistics with only a small data rate loss but has no inherent error control. In the PTS scheme, OFDM subcarriers subblocks are phase shifted separately after the inverse fast Fourier transform (IFFT) computation. It has been demonstrated that if the subblocks are optimally phase shifted, they result in minimum PAPR and consequently reduce the PAPR of the combined signal. In the PTS scheme, the number of subblocks and their partitioning determine the extent of PAPR reduction. Nevertheless, this approach still needs many IFFTs and also the number of optional phase factor sequences is very large. Hence it is very difficult to determine which phase factor sequences are employed in the optimization process $[15,16]$. To alleviate the problem of high complexity an approach [17] has been proposed, in which real and imaginary parts are separately multiplied with phase factors, moreover PAPR is conjointly optimized in real and imaginary parts. This scheme has lower complexity with degraded PAPR performance compared to conventional PTS. Coding techniques have been utilized to correct errors as well as to control the PAPR. The combined forward error-correcting code (FECs) with PTS technique [18, 19] for a QPSK modulation has also been proposed. This method divides data bits into PAPR control bits and information bits and then uses these PAPR bits to generate a number of candidates to represent the same OFDM signal [20-22]. This requires a complex optimization process, particularly when large number of subcarriers is employed. Hence the modified PTS with forward error correcting codes such as Golay complementary sequences with Reed-Muller code and turbo code is proposed to provide better PAPR reduction and lower computational complexity in this paper.

The rest of the paper is organized as follows: Section 2, briefly introduces PAPR in OFDM system. Section 3, outlines the definition of complementary Golay sequences and Reed-Muller code and their properties. Turbo code with PTS scheme is presented in Section 4. Section 5 presents the simulation results. And finally, conclusions are drawn in Section 6.

\section{PAPR IN OFDM SYSTEM}

Considering, $\mathrm{N}$ modulated data symbols from a particular signaling constellation, to create a complex-valued symbol vector $X_{k}=X_{0}, X_{1}, \ldots . X_{N-1}$,

where, $X_{k}$ is the complex value carried by the $\mathrm{k}^{\text {th }}$ subcarrier.

The OFDM symbol can be written as

$x \quad t=\frac{1}{\sqrt{N}} \sum_{k=0}^{N-1} X_{k} e^{j 2 \pi k f_{0} t} \quad, \quad 0 \leq t \leq T$

where $\mathrm{T}$ is the symbol interval, and $f_{0}=1 / \mathrm{T}$ is the frequency spacing between adjacent subcarriers.

Replacing $\mathrm{t}=\mathrm{n} T_{b}$, where $T_{b}=\mathrm{T} / \mathrm{N}$, gives the discrete time version denoted by

$x(n)=\frac{1}{\sqrt{N}} \sum_{k=0}^{N-1} X_{k} e^{j 2 \pi k n / L N}, \mathrm{n}=0,1, \ldots \mathrm{NL}-1$

where, L is the oversampling factor. The symbol-spaced sampling sometimes misses some of the signal peaks and results 
in optimistic results for the PAPR. The sampling can be implemented by an inverse fast Fourier transform (IFFT).

The PAPR of the signal, $x(t)$, is then given as the ratio of the peak instantaneous power to the average power, written as

$P A P R=\frac{\max _{0 \leq t \leq T}|x(t)|^{2}}{\left.E\left[\mid \begin{array}{ll}x & t^{2}\end{array}\right]\right]}$

where $E[\cdot]$ is the expectation operator.

From the central limit theorem, for large values of $\mathrm{N}$, the real and imaginary values of $x(t)$ becomes Gaussian distributed. The amplitude of the OFDM signal, therefore, has a Rayleigh distribution with zero mean and a variance of $\mathrm{N}$ times the variance of one complex sinusoid. The complementary cumulative distribution function (CCDF) is the probability that the PAPR exceeds a certain threshold $P A P R_{0}$.

$C C D F(P A P R(x(n)))=\mathrm{P}_{r}(\operatorname{PAPR}(x(n)))>P A P R_{0}$

Due to the independence of the $\mathrm{N}$ samples, the CCDF of the PAPR of a data block with Nyquist rate sampling is given by

$$
P=\mathrm{P}_{r}\left(\operatorname{PAPR}(x(n))>P A P R_{0}\right)=1-\left(e^{-P A P R_{0}}\right)^{N}
$$

This expression assumes that the $\mathrm{N}$ time domain signal samples are mutually independent and uncorrelated and it is not accurate for a small number of subcarriers. Therefore, there have been many attempts to derive more accurate distribution of PAPR.

\section{GOLAY SEQUENCES AND REED- MULLER CODE \\ 3.1 Coding Theory}

The base of coding scheme is to reduce the OFDM PAPR. PAPR can be reduced by using a selected code, which only produces OFDM symbols for which the PAPR is below the desirable level. There exist a large number of code words suitable for coding scheme. But it requires a structured way of encoding and decoding to generate these code words, and the code properties. Golay complementary sequences provide the way for generating PAPR reduction codes. Golay complementary sequences are the sequence pairs for which the sum of autocorrelation functions is zero for all delay shifts unequal to zero. This correlation property of complementary sequences translates into a relatively small PAPR of $3 \mathrm{~dB}$ when the codes are applied to modulate the OFDM signal.

\subsection{Complementary Sequence Theory}

A sequence $x$ of length $\mathrm{N}$ is said to be complementary to another sequence $y$ if the following condition holds on the sum of both autocorrelation functions,

$$
\begin{aligned}
\sum_{k=0}^{N-1}\left(x_{k} x_{k+i}+y_{k} y_{k+i}\right) & =2 N ; & & i=0 \\
& =0 ; & & i \neq 0
\end{aligned}
$$

After taking the Fourier transform on both sides of Eq. (6) the above condition is translated into the following equation.
$|X(f)|^{2}+|Y(f)|^{2}=2 N$

$|X(f)|^{2}$ is the power spectrum of $x$, which is the Fourier transform of its autocorrelation function. From Eq. (7), it is observed that the maximum value of the power spectrum is bounded by $2 \mathrm{~N}$.

$|X(f)|^{2} \leq 2 N$

Because the average power of $\mathrm{X}(\mathrm{f})$ is equal to $\mathrm{N}$, assuming that the power of the sequence $x$ is equal to 1 , the PAPR of $\mathrm{X}(\mathrm{f})$ is bounded as

$P A P R \leq \frac{2 N}{N}=2 \quad(=3 \mathrm{~dB})$

The PAPR upper bounded by 2 is valid when X(f) is replaced by the inverse Fourier transform of the sequence, $x$. By using a complementary code as input to generate an OFDM signal, it is guaranteed that the PAPR doesn't exceed $3 \mathrm{~dB}$ [11].

The large sets of binary complementary pairs of length $2^{\mathrm{m}}$ can be obtained from the $2^{\text {nd }}$ order co-sets of the well-known $1^{\text {st }}$ order Reed-Muller code $\mathrm{R}(1, \mathrm{~m})$. This Reed-Muller code results in low PAPR in addition to its error-correcting capability.

The $\mathrm{r}^{\text {th }}$ order Reed-Muller code is designated as $\mathrm{R}(\mathrm{r}, \mathrm{m})$, where $\mathrm{m}$ is the parameter related to the length of the code, $n=2^{m}$, and $0 \leq \mathrm{r} \leq \mathrm{m}$. Half of the codes of $\mathrm{R}(\mathrm{r}, \mathrm{m})$ are complements of the other half. $\mathrm{R}(1, \mathrm{~m})$ is also known as a bi-orthogonal code since it can be obtained from the generator matrix of an orthogonal code by adding all-ones codeword to it [12].

PTS is based on combining signal sub blocks which are phased-shifted by different phase factors to generate multiple candidate signals, so as to select the low PAPR signal. In general, the procedure for PTS is obtained as following.

First, consider the data block, $X=X_{0}, X_{1}, \ldots \ldots X_{N-1}$. Encode the data block by using Reed Muller code. Define the codeword as a vector, $S=C_{0}, C_{1}, \ldots . . C_{N-1}{ }^{T}$, where, $c$ is an encoded data such as Reed Muller code.

Secondly, $\mathrm{S}$ to be transmitted is divided into several subblocks, V, by using subblock partition scheme. In general, subblock partition scheme can be classified into 3 categories. The three partition methods are adjacent, interleaved and random.

$\mathrm{S}$ is partitioned into $M$ disjoint sets, which is represented by the vector,

$S_{m}, m=1,2, . ., M$

In this work, the codeword vector $S$ is partitioned by using adjacent method. Assume that the subblocks or clusters consist of a contiguous set of subcarriers and are of equal size. The objective is to optimally combine the $M$ clusters, which in frequency domain is given by

$S^{\prime}=\sum_{m=1}^{M} b_{m} S_{m}$

where, $\left\{b_{m}, m=1,2, \ldots, M\right\}$ are weighting factors and are assumed to be perfect rotations.

In other words, the time domain is given by 


$$
s=\sum_{m=1}^{M} b_{m} s_{m}
$$

where, $S_{m}$ which is called the partial transmit sequence, is the IFFT of $S_{m}$. The phase factors $b_{m}$ are chosen to minimize the PAPR of s'. By using 256 subcarriers, $M=4$, Peak to Average Power Ratio (PAPR) is reduced from $1 \%$ to more than $3 \mathrm{~dB}$. PTS generates a signal with a low PAPR through the addition of appropriately phase rotated signal parts. The codeword to be transmitted are divided into several subblocks, V, of length N/V. Mathematically, expressed by

$A_{k}=\sum_{v=1}^{V} A_{k}^{\left({ }^{(v)}\right.}$

All subcarriers positions in $A_{k}^{(v)}$ which are occupied in another subblock are set to zero. Each of the blocks, $v$, has an IFFT performed on it,

$a_{n}^{(v)}=\operatorname{IFFT} A_{k}^{(v)}$

The output of each block (except for first block which is kept constant) is phase rotated by the rotation factor as given by

$e^{j \theta(v)} \in[0,2 \pi]$

The blocks are then added together to produce alternate transmit signals containing the same information as given by

$\tilde{a}_{n}=\sum_{v=1}^{V} a_{n}^{(v)} \cdot e^{j \theta(v)}$

Each alternate transmit signal is stored in memory and the process is repeated again with a different phase rotation value. After a set number of phase rotation values, $W$, the OFDM symbol with the lowest PAPR is transmitted as given by

$$
\tilde{\phi}^{2}, \tilde{\phi}^{3}, \ldots . \tilde{\phi}^{v}=\arg \min \left(\max \left|\tilde{a}_{n}\right|\right)
$$

The weighting rotation parameter set is chosen to minimize the PAPR. The computational complexity of PTS method depends on the number of phase rotation factors allowed. The phase rotation factors can be selected from an infinite number of phases $\phi^{(v)} \in(0,2 \pi)$. But finding the best weighting factors is indeed a complex problem.

By using multicarrier modulation technique, the crosstalk between the subcarriers should be minimized. So it is required to maintain the orthogonality between the different modulated carriers. The basic idea of PTS is to combined signal subblocks which are phased-shifted by different phase factors to generate multiple candidate signals, so that the phase factor that results in low PAPR can be selected. These phase factors combination correctly maintain the orthogonality between the different modulated carriers. The coding method adds pattern of redundancy to the input data in order to reduce the PAPR.

\section{TURBO CODING}

In this work, a turbo encoder is employed which offer two advantages, significant PAPR reduction and good bit error rate performance. Figure 1 shows the block diagram of turbo encoder. Turbo codes [15] are parallel concatenated convolutional codes in which the information bits are first encoded by a recursive systematic convolutional (RSC) code and then, after passing the information bits through an interleaver, are encoded by a second RSC code. The purpose of interleaving the coded data transforms burst error into independent errors. The result of interleaving makes error burst to spread out in time, so that errors within a codeword appear to be independent. The role of puncture is to periodically delete the selected bits to reduce the coding overhead Turbo decoder is used to recover the transmitted signal at the receiver side.

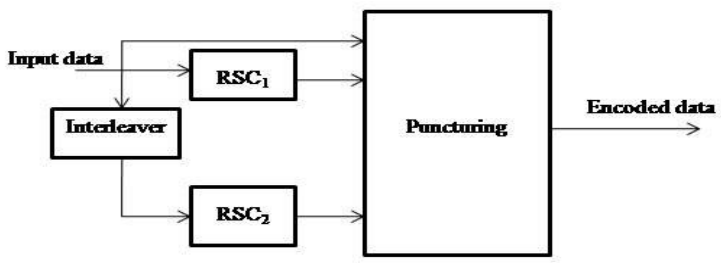

Figure 1 Turbo encoder

Also the turbo encoder can be used to generate different sequences and sequence with lowest PAPR is selected for transmission. Figure 2 shows the transmitter side of an OFDM system, where the turbo coding and PTS are used for PAPR reduction.

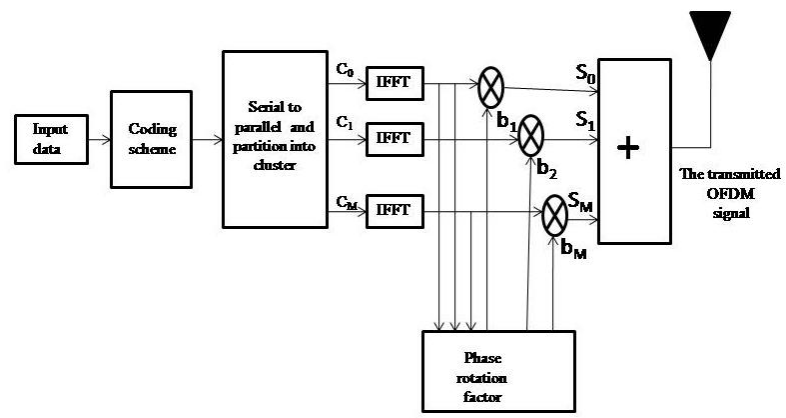

Figure 2 Block diagram of system model

The procedure for turbo PTS is same as Golay PTS except ReedMuller complement sequence is replaced with turbo encoder sequence. So by combining these two methods, significant performance improvement can be achieved.

\section{RESULTS AND DISCUSSION}

The analysis of the modified PTS with forward error correcting codes such as Golay complementary sequences with Reed-Muller code and turbo code techniques has been done using MATLAB 7.0. The simulation parameters considered for this analysis is summarized in Table 1.

Table 1.Simulation parameters

\begin{tabular}{|c|c|}
\hline Simulation parameters & Type/Value \\
\hline Number of subcarriers & $64,128,256,512,1024$ \\
\hline Number of subblocks & $2,4,8,16$ \\
\hline Oversampling factor & 4 \\
\hline Modulation Scheme & QPSK \\
\hline Phase factor & $1,-1, \mathrm{j},-\mathrm{j}$ \\
\hline
\end{tabular}




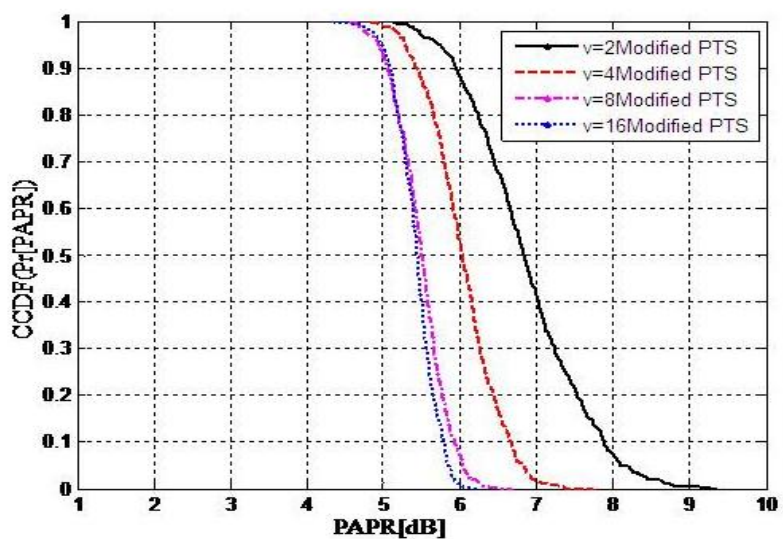

Figure 3 Modified PTS performance for various subblocks $\mathrm{V}=2, \mathbf{4}, \mathbf{8 ,} 16$ with $\mathrm{N}=256$

For comparison, PAPR reduction techniques with phase rotation factor and combined FEC-PTS with phase rotation are deemed. In the OFDM system under consideration, modified PTS technique is applied to the subblocks of uncoded information, which is modulated by QPSK, and the phase rotation factors are transmitted directly to receiver through subblock. The performance evaluation is done in terms of complementary cumulative distribution function.

Figure 3 shows the performance of modified PTS for various subblocks for a random data of block size 1000 with $\mathrm{N}=256$. It is noted that at CCDF 0.6 while the subblock size is increased from 2 to 4,8 , and 16 , the PAPR is reduced by $0.8 \mathrm{~dB}, 1.3 \mathrm{~dB}$ and 1.4 $\mathrm{dB}$ respectively form $6.7 \mathrm{~dB}$, which signifies the improvement in the performance.

Figure 4 illustrates the performance of modified PTS for subblock size $\mathrm{V}=4$ for various subcarriers. Significant performance gain is seen for $\mathrm{N}=64$ compared with $\mathrm{N}=128,256$, 512, and 1024, an improvement of $0.7,1.4,2$, and $2.6 \mathrm{~dB}$ respectively in PAPR can be attained for CCDF of 0.6.

Figure 5 illustrates the performance of combined Golay with PTS for subblock size $\mathrm{V}=4$ for various subcarriers $\mathrm{N}=64,128,256$, 512, and 1024.It is observed from the figure even if the number of subcarriers increases there is no significant change in PAPR since for any Golay sequences the PAPR value is at most $3 \mathrm{~dB}$.

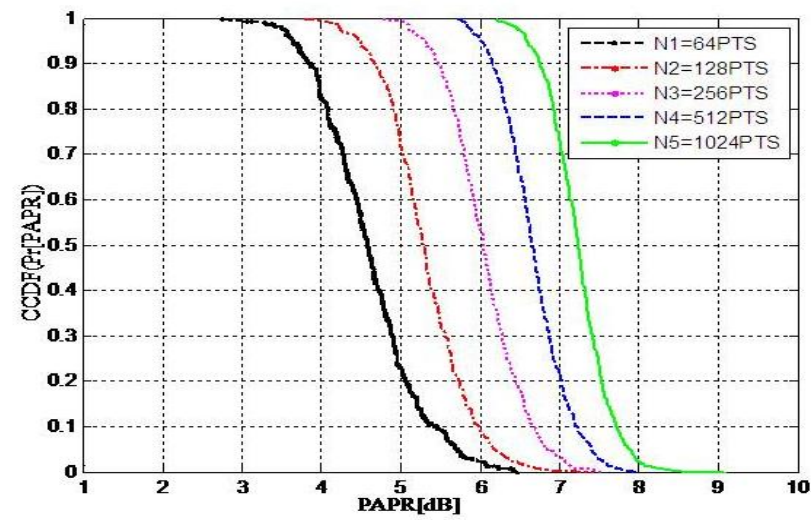

Figure 4 Modified PTS performance for subblock $V=4$ with for various subcarriers $N=64,128,256,512,1024$

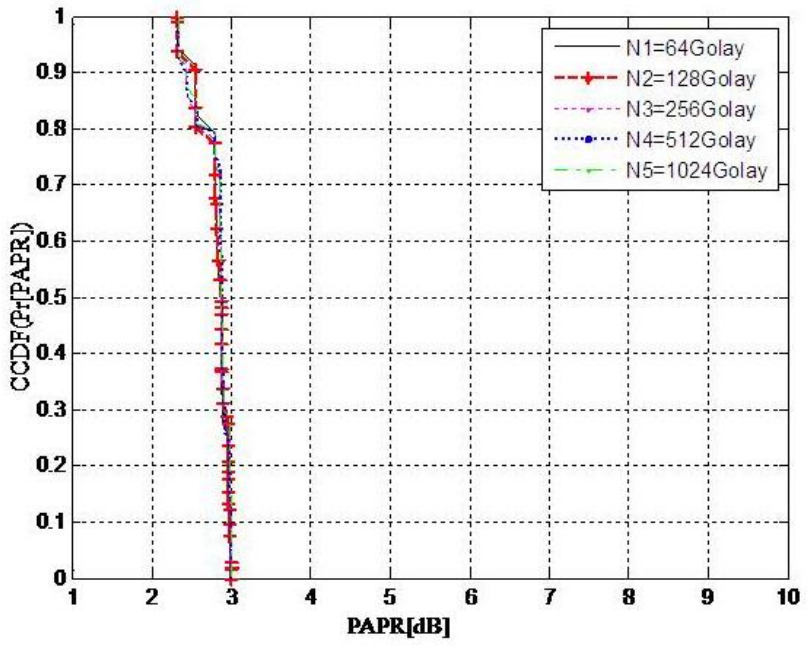

Figure 5 PAPR reduction using PTS combined with Golay sequence when $V=4$ with subcarriers $N=64,128,256,512$ and 1024

Referring to Figure 4 and Figure 5 it can be inferred that, combined Golay with PTS results in significant performance gain in terms of PAPR reduction compared to a scheme without FEC. Furthermore for CCDF of 0.6 with $\mathrm{N}=1024$, PAPR of Golay complementary sequence reduces by $4 \mathrm{~dB}$ as compared to uncoded scheme.

In Figure 6, the combined scheme is evaluated for various subblocks $\mathrm{V}=2,4,8$, and 16 . It is observed from the figure for any subblock length the PAPR is always lies around $3 \mathrm{~dB}$ values. Furthermore for CCDF of 0.6 with V=16, PAPR of Golay complementary sequence reduces by $3 \mathrm{~dB}$ as compared to uncoded scheme.

Figure 7 shows the performance results of modified PTS with Turbo coding for subblock size $\mathrm{V}=4$ for various subcarriers. Here, also $\mathrm{N}=64$ results in better PAPR reduction compared to $\mathrm{N}=1024$. However, as $\mathrm{N}$ is increased from 64 to 128, 256, 512 and 1024 the PAPR is increased to $0.8,1.8,2.3$, and $3 \mathrm{~dB}$ from $3.6 \mathrm{~dB}$ for CCDF of 0.6.

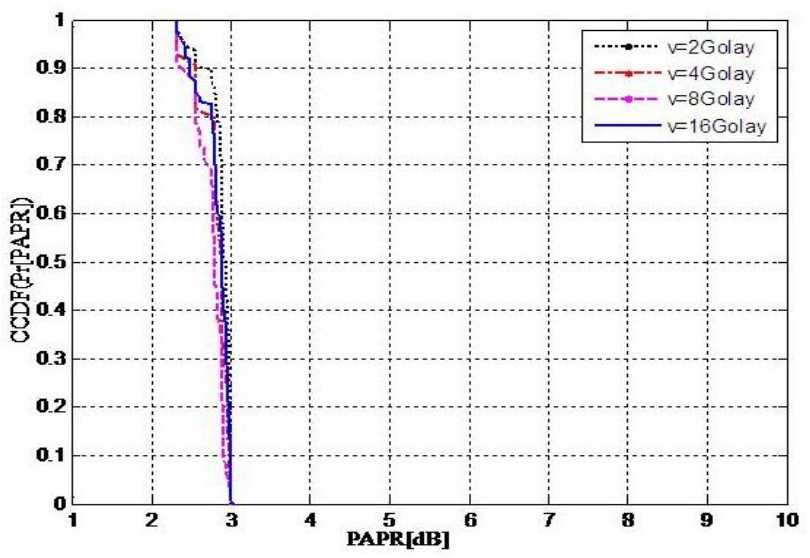

Figure 6 PAPR reduction using PTS combined with Golay sequence in OFDM when $\mathrm{N}=256$ with subblocks $\mathrm{V}=2,4,8$ and 16 


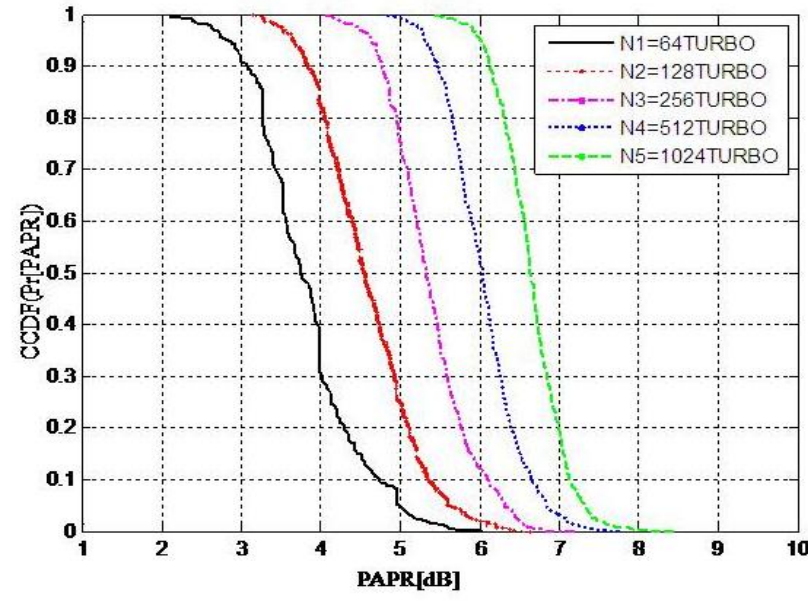

Figure 7 PAPR reduction using PTS combined with turbo codes in $O F D M$ when $V=4$ with subcarriers $N=64,128,256$, 512 and 1024

In Figure 8 the combined scheme is investigated for various subblocks size. The result shows subblock size $V=4,8$, and 16 provides an improvement of $0.8,2$, and $3 \mathrm{~dB}$, respectively compared to $\mathrm{V}=2$. Therefore it can be concluded that for Turbo PTS technique, it optimal to choose $\mathrm{V}=16$ with $\mathrm{N}=256$ to achieve considerable performance gain.

Figure 9 demonstrates the performance comparisons of an OFDM system with and without PAPR reduction techniques. Here, modified PTS, Turbo PTS and Golay PTS PAPR reduction techniques are considered for performance comparisons. The combined Golay PTS scheme using adjacent partition method result in almost the same PAPR reduction while keeping low complexity. And, also performance gain of around $2.4 \mathrm{~dB}$ and $3 \mathrm{~dB}$ for $\mathrm{CCDF}$ value of 0.6 , the subblock size of $\mathrm{V}=4$ and a random data of block size 1000 with QPSK modulation with $\mathrm{N}=256$ is achieved compared to Turbo PTS and modified PTS respectivly.

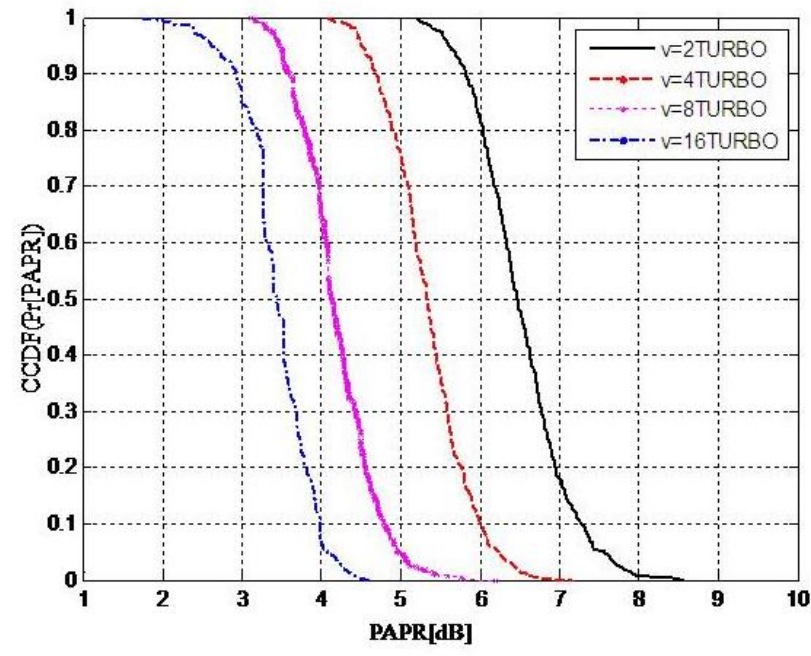

Figure 8 PAPR reduction using PTS combined with turbo codes in OFDM when $\mathrm{N}=256$ with subblocks $\mathrm{V}=2, \quad 4,8$ and 16

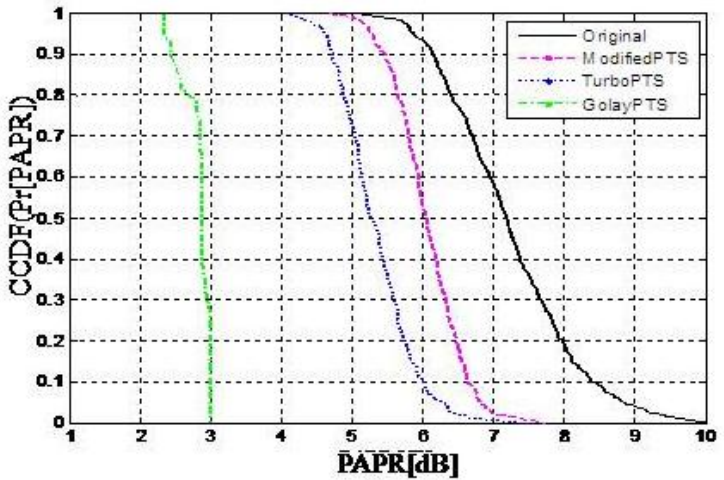

Figure 9 PAPR reduction using PTS with Golay sequence and Turbo codes in OFDM system

\section{CONCLUSION}

In this paper, PAPR reduction technique based on modified PTS with FEC in OFDM systems is considered. This approach, which combines the PTS technique with Golay complementary sequences and Reed-Muller code, divides the subcarriers of OFDM into several disjoint subblocks resulting in significant performance gain in terms of PAPR reduction with low complexity. Turbo PTS provides good PAPR performance but as the number of subcarrier increases PAPR increases. Golay PTS results in a performance improvement of $2.3 \mathrm{~dB}$ in terms of PAPR reduction compared to Turbo PTS for $\mathrm{N}=256$ and a CCDF value of 0.6 and also provides low complexity.

\section{REFERENCES}

[1] S.H.Muller and J.B.Huber., 1997. A novel peak power reduction scheme for OFDM. In Proceedings of IEEE International Symposium on Personal, Communication (PIMRC), 1090-1094.

[2] M.Pauli and H.P.Kuchenbecher., (1998). On the reduction of the out of band radiation of OFDM signals. In Proceedings of IEEE International Conference on Communications, (June 1998), 1304-1308.

[3] X.Li and L.J.Cimini., (1998). "Effect of clipping and filtering on the performance of OFDM". IEEE Communications Letters, (May 1998), 131-133.

[4] H.Ochiai and H.Imai., (2002). "Performance analysis of deliberately clipped OFDM signals", IEEE Transactions on Communications, (Jan.2002), 89-101.

[5] H.Saeedi, M.Sharif, and F.Marvasti., (2002). "Clipping noise cancellation in OFDM system using oversampled signal reconstruction", IEEE Communications Letters, (Feb.2002), 73-75.

[6] X.Wang, T.T.Tjhung, and C.S.Ng., (1999). "Reduction of peak-to-average power of OFDM system using a companding technique", IEEE Transactions on Broadcasting, (Sep.1999), 303-307.

[7] X.Huang, J.Lu, J.Zheng, and J.Chuang.,(2001). "Reduction of peak-to-average power ratio of OFDM signals with companding transform”, Electronics Letters, (April 2001) 506-507.

[8] M.Sharif, M.Gharayi-Alkhansari, and B.H.Khalaj., (2003). "On the peak-to-average power of OFDM signals based on 
oversampling", IEEE Transactions on Communications, (Jan.2003), 72-78.

[9] X. Huang, J. Lu, 7. Zheng, K. B. Letaief, and J. Gu., (2004). "Companding transform for reduction in peak-to-average power of OFDM signals", IEEE Transaction on Wireless Communications, (Nov. 2004), 2030-2039.

[10] T.A.Wilkinson and A. E. Jones.,(1995). Minimization of the peak to mean envelope power ratio in multicarrier transmission schemes by block coding. In Proceedings of IEEE Vehicular Technology Conference, (July 1995), $825-829$.

[11] J.A.Davis and J.Jedwab.,(1999). "Peak to mean power control in OFDM, Golay complementary sequences, and Reed-Muller codes", IEEE Transactions on Information Theory, (Nov. 1999), 2397-2417.

[12] K.Patterson.,(2000). "Generalized Reed-Muller codes and power control in OFDM modulation", IEEE Transactions on Information Theory, (Jan. 2000), 104-120.

[13] A.D.S.Jayalath and C.Tellambura., (2000). "Adaptive PTS approach for reduction of peak-to-average power ratio of OFDM signals", Electronics Letters, (July 2000), 226-1228.

[14] M.Breiling, S.H.Muller, and J.B.Huber., (2001). "SLM peak-power reduction without explicit side information", IEEE Communications Letters,(June 2001), 239-241.

[15] Osamu Muta and Yoshihiko Akaiwa, 2008. Iterative weighting factor estimation method for peak power reduction with adaptive subcarrier-phase control in Turbo-coding multi-carrier CDM systems, in proceedings of IEEE $68^{\text {th }}$ Vehicular Technology Conference(VTC'08), 1-5.

[16] A.D.S.Jayalath and C.Tellambura.,(2005)."SLM and PTS peak-power reduction of OFDM signals without side information", IEEE Transactions on Wireless Communications, (Sep. 2005), 2006-2013.

[17] Xinchun Wu, Zhigang Mao, Jinxiang Wang, Bin Zho.2009. A novel PTS technique with combinative optimization in real part and imaginary part for PAPR reduction in OFDM systems. In proceedings of Third International Conference on Next Generation Mobile Applications, Services and Technologies, 215-218.

[18] Abolfazl Ghassemi, T.Aaron Gulliver.,(2010), "PAPR reduction of OFDM using PTS and error-correcting code subblocking", IEEE Transactions on wireless communications, (March 2010), 980-989.
[19] Fangling PU, Jianya Gong, Liangcai Gan., 2005. OFDM peak-to-average power ratio reduction by combining the PTS with Golay complementary sequences and Reed-Muller codes. In proceedings of International Conference on Advanced Communication Technology. 845-848.

[20] A.D.S.Jayalath and C.Tellambura., (2000). The use of interleaving to reduce the peak to average power ratio of an OFDM signals. IEEE Global Telecommunications Conference (December 2000), 82-86.

[21] V.Tarokh and H.Jafarkhani.,(2000).“On the computation and reduction of the peak-to-average power ratio in multicarrier communications", IEEE Transactions on Communications, (January 2000), 37-44.

[22] Lin Yang., 2008.Link-level enhancement schemes utilizing multiple symbol representation for Multicarrier communication. Ph.D. thesis, University of Manchester.

\section{AUTHORS PROFILE}

P. Mukunthan received B.E degree in Electronics and Communication Engineering from University of Madras, Chennai in 1998 and M.E degree in Applied Electronics from University of Madras, Chennai in 2000. He is working as Assistant Professor in the Department of Computer Science and Engineering, Adhiparasakthi Engineering College, Melmaruvathur, India. He is pursuing his Ph.D. in the Department of Electronics and Communication Engineering, Pondicherry Engineering College, Pondicherry. His research interests include digital communication, computer networks, wireless cellular mobile network.

P. Dananjayan received Bachelor of Science from University of Madras in 1979, Bachelor of Technology in 1982 and Master of Engineering in 1984 from the Madras Institute of Technology, Chennai and Ph.D. degree from Anna University, Chennai in 1998. He is working as Professor in the Department of Electronics and Communication Engineering, Pondicherry Engineering College, Pondicherry, India. He has been as visiting professor to AIT, Bangkok. He has to his credit more than 75 publications in National and International Journals. He has presented more than 160 papers in National and International Conferences. He has guided $9 \mathrm{Ph} . \mathrm{D}$ candidates and is currently guiding $6 \mathrm{Ph} . \mathrm{D}$ students. His research interests include spread spectrum techniques, wireless communication, wireless adhoc and sensor networks. 